\title{
Formation of Long-Term Memory Representation in Human Temporal Cortex Related to Pictorial Paired Associates
}

\author{
Ken-ichiro Yamashita, ${ }^{1}$ Satoshi Hirose, ${ }^{1}$ Akira Kunimatsu, ${ }^{2}$ Shigeki Aoki, ${ }^{2}$ Junichi Chikazoe, ${ }^{1}$ Koji Jimura, ${ }^{1}$ \\ Yoshitaka Masutani, ${ }^{2}$ Osamu Abe, ${ }^{2}$ Kuni Ohtomo, ${ }^{2}$ Yasushi Miyashita, ${ }^{1}$ and Seiki Konishi ${ }^{1}$ \\ Departments of ${ }^{1}$ Physiology and ${ }^{2}$ Radiology, The University of Tokyo School of Medicine, 7-3-1 Hongo, Bunkyo-ku, Tokyo 113, Japan
}

It is widely held that long-term memory gradually develops in the temporal neocortex after initial memory encoding into the hippocampus. However, little is known as to whether and where long-term memory can be newly created in the human temporal neocortex. In this functional magnetic resonance imaging study, we detected brain activity in the temporal neocortex that was developed $\sim 8$ weeks after study of unfamiliar pictorial paired associates. Two sets of paired Fourier figures were studied, one $\sim 8$ weeks before test and the other immediately before test, keeping the correct performance during the tests balanced across the two sets of stimuli. Significant signal increase was observed in the right hippocampus during retrieval of newly studied pairs relative to initially studied pairs. In contrast, significant signal increase was observed in the anterior temporal cortex during retrieval of initially studied pairs relative to newly studied pairs. The greater activity during retrieval of older memory developed in the temporal neocortex provides direct evidence of formation of temporal neocortical representation for stable long-term memory.

\section{Introduction}

Since the initial report on patient H.M. (Scoville and Milner, 1957), the medial temporal lobe (MTL) has been a focus of investigation concerning long-term memory (Mishkin and Murray, 1994). Memory-related deficits induced by the damage to the MTL (Milner et al., 1968) or the hippocampus proper (Zola-Morgan et al., 1986) include anterograde and retrograde amnesia. The retrograde amnesia has been characterized by subsequent studies that demonstrated a temporally graded role of the hippocampus proper in memory retrieval (Zola-Morgan and Squire, 1990). These neuropsychological and experimental approaches converged to a theory of memory consolidation: Long-term memory initially retrieved via the hippocampus is gradually reorganized in the neocortex and becomes independent of the hippocampus (Squire and Alvarez, 1995). Subsequent studies further showed that the consolidation occurs during slow-wave sleep (Plihal and Born, 1997; Takashima et al., 2006; Gais et al., 2007; Yoo et al., 2007). On the other hand, an alternative theory, called multiple trace theory, has also been proposed: long-term memory traces in the hippocampus and the neocortex are multiplied, but retrieval continues to depend on the hippocampus (Nadel and Moscovitch, 1997). Although the role of the hippocampus in long-term memory formation differs in these theories, development of the temporal neocortical representation is consistently predicted.

Received March 17, 2009; revised May 26, 2009; accepted July 2, 2009.

This work was supported by a Grant-in-Aid for Specially Promoted Research (19002010) to Y.M., by a grant (17500203) to S.K. from the Ministry of Education, Culture, Sports, Science, and Technology, Japan, by a Research Fellowship (195708) to K.Y. from the Japan Society for the Promotion of Science for young scientists, and by Takeda Science Foundation (Y. M.).

Correspondence should be addressed to either Dr. Seiki Konishi or Prof. Yasushi Miyashita, Department of Physiology, The University of Tokyo School of Medicine, 7-3-1 Hongo, Bunkyo-ku, Tokyo 113, Japan, E-mail: konishi@m.u-tokyo.ac.jp or yasushi_miyashita@m.u-tokyo.ac.jp.

D0I:10.1523/JNEUROSCI.1328-09.2009

Copyright $\odot 2009$ Society for Neuroscience $\quad 0270-6474 / 09 / 2910335-06 \$ 15.00 / 0$
The neural correlates of memory representation have been investigated in studies of nonhuman primates using electrophysiology, and a population of neurons has been found to be activated in the inferior temporal (IT) cortex during performance of a pair-association (PA) task (Sakai and Miyashita, 1991; Naya et al., 2003). A subsequent combined lesion and electrophysiology study further showed that the response of the IT neurons was dependent on the MTL, suggesting the interaction between the MTL and the IT neurons during formation of memory representation (Higuchi and Miyashita, 1996; Messinger et al., 2001). Whereas the role of the hippocampus in long-term memory formation in humans have also been investigated using noninvasive neuroimaging techniques (Stark and Squire, 2000; Haist et al., 2001; Takashima et al., 2006; Smith and Squire, 2009), the neocortical stores for more enduring memory representation largely remain to be explored.

In this study, we used functional magnetic resonance imaging (fMRI) to identify the neural substrates for memory representation in the temporal neocortex developed after learning of pictorial stimuli. Subjects initially studied a set of Fourier figures by performing the PA task. Approximately 8 weeks after study, the subjects studied another new set of figures. The 8 week delay is consistent with results of a previous study on memory formation (Zola-Morgan and Squire, 1990). Importantly, performance of these two sets of stimuli was kept balanced even after the long delay of 8 weeks by making the number of exposures in the initial study much greater than that in the subsequent study. After study of the two sets of pairs, fMRI was used to compare the brain activity during retrieval of the stimuli learned long before the test (remote memory) with that during retrieval of newly learned stimuli (recent memory), to identify the neural substrates associated with representation of initially learned words. 


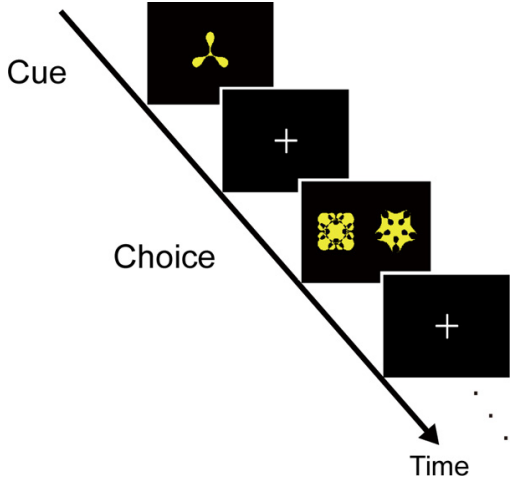

Figure 1. PA task. The PA task consisted of cue and choice periods in which a cue was presented and then a paired associate had to be selected out of two alternatives, a target and a distractor.

\section{Materials and Methods}

Subjects. Written informed consent was obtained from 30 healthy right-handed subjects (18 males, 12 females; age, 20-27). They were scanned using experimental procedures approved by the institutional review board of the University of Tokyo School of Medicine.

fMRI procedures. The experiments were conducted using a 3T fMRI system. Scout images were first collected to align the field of view centered on the subject's brain. Fast spin-echo images were obtained for anatomical reference $[$ repetition time $(\mathrm{TR})=3000 \mathrm{~ms}$; echo time $(\mathrm{TE})=$ $85 \mathrm{~ms} ; 80$ slices, slice thickness $=2.0 \mathrm{~mm}$; in-plane resolution $=1 \times 0.67$ $\mathrm{mm}]$. For functional imaging, a gradient echo echo-planar sequence was used $(\mathrm{TR}=3000 \mathrm{~ms} ; \mathrm{TE}=35 \mathrm{~ms}$; flip angle $=90$ degrees; $40 \times 4 \mathrm{~mm}$ slices; in-plane resolution of $4 \times 4 \mathrm{~mm}$ ). One functional run was collected, and the first four functional images were excluded from the analysis to take into account the equilibrium of longitudinal magnetization.

Task. The PA task had a structure that resembled those used in our previous monkey electrophysiological studies (Sakai and Miyashita, 1991; Naya et al., 2003) (Fig. 1). The PA task began with the presentation of a Fourier figure (Naya et al., 2003) as a cue stimulus (cue period), which was followed by the presentation of two figures as a target and a distracter, one to the right and the other to the left (choice period). The figure presented as a cue was not presented as a target or a distractor. The PA task had two variants administered in study and test sessions that differed in time parameters and presence/absence of feedback stimuli (O: correct, $\mathrm{X}$ : incorrect). The task sequence in the study session was as follows: cue period for $1 \mathrm{~s}$, fixation gap for $0.5 \mathrm{~s}$, choice period for $2.5 \mathrm{~s}$, fixation gap for $0.5 \mathrm{~s}$, feedback period for $0.5 \mathrm{~s}$, intertrial interval for $3.0 \mathrm{~s}$. On the other hand, the task sequence in the test session was as follows: cue period for $0.5 \mathrm{~s}$, fixation gap for $0.5 \mathrm{~s}$, choice period for $2.5 \mathrm{~s}$, intertrial interval for $0.5 \mathrm{~s}$, without presentation of feedback stimuli.

Schedule. The subjects underwent study session 1 (S1), study session 2 (S2), and the test session (Fig. 2). Before S1 and S2, 20 unfamiliar Fourier figures had been presented twice each for $4 \mathrm{~s}$ to familiarize the subjects with the figures. S1 consisted of learning of 10 pairs of the figures [round 1 (R1)] and relearning of the same 10 pairs [round 2 (R2)] that were separated by $\sim 2$ weeks. S2 was separated from R2 in $\mathrm{S} 1$ by $\sim 8$ weeks, during which the formation of memory representation for the learned stimuli was expected to take place, and the subjects learned a new set of 10 pairs in S2. These two sets of 10 pairs were counterbalanced across subjects, such that one set of pairs presented in S1 in one subject was presented in S2 in another. To make performance of retrieval of remote and recent memory in the test session balanced, different learning criteria were applied to S1 and S2. The learning in S1 was administered persistently, and both R1 and R2 in S1 continued until they achieved $100 \%$ correct performance in three successive blocks of 20 trials in which each of the 10 pairs was presented twice. On the other hand, the learning in S2 was made shallower by using the criteria of $70 \%$ correct in one block of 10 trials in which each of the 10 pairs was presented once.
After completing S2, the test session was administered $\sim 30$ min later while functional images were acquired (Fig. 2). Ten PA trials in which the 10 pairs from S1 (remote trials) were presented and another 10 PA trials in which the 10 pairs from S2 (recent trials) were presented, together with fixation trials used for the event-related fMRI design, were pseudorandomly intermixed within an fMRI run.

Confidence scores were collected for each of remote and recent trials after completing the scan. The subjects were presented with the same intact PA pairs in the same order, and were asked to provide the confidence score for each PA pair. The validity of the scoring procedure was later confirmed (see Results, Behavioral data). The score was rated 3 when the subjects evaluated that the PA trial would be correct by $90 \%$ or more. Similarly, the score was rated 2 when the subjects evaluated that the PA trial would be correct by $\sim 70 \%$, and the score was rated 1 when the subjects judged that the PA trial would be correct by chance $(\sim 50 \%$ correct).

To obtain behavioral measures that may reflect stable long-term memory representation for the learned pairs, before the start of R2 in S1, a mid-session retention test was administered in which the 10 pairs learned in R1 in $\mathrm{S} 1$ were tested. The task sequence and the time parameters in the PA trials were the same as those used in the test session, to compare reaction times in the two tests administered $\sim 8$ weeks apart. Note that the same pairs of the Fourier figures were presented in remote trials in the test session and all the trials in the mid-session retention test, but that the same pairs were tested in the test session and the mid-session retention test $\sim 8$ and 2 weeks after learning, respectively. The reaction time difference in each subject was used for a subsequent correlation analysis made based on the following logic: The greater the brain activity in the temporal neocortex that reflects the stable long-term memory representation formed during the long interval, the shorter the reaction time in remote trials in the test session relative to that in the mid-session retention test.

Data analysis. Data were analyzed using SPM2 software (http://www. fil.ion.ucl.ac.uk/spm/). Functional images were realigned, slice timing was corrected, normalized to the baseline template with interpolation to a $2 \times 2 \times 2 \mathrm{~mm}$ space, and spatially smoothed (full width at half maximum $=10 \mathrm{~mm})$. Then event timing was coded into a general linear model (Worsley and Friston, 1995). Events at the time of the correct remote trials with the confident score 2 or 3 and the correct recent trials with the confident score 2 or 3 , together with other events of no interest such as error trials, were modeled as events, time-locked to the onset of cue stimulus presentation using the canonical function in SPM2. Correct trials with no confidence (score: 1 ) were included in the regressor that coded error trials.

The levels of confidence in remote and recent trials that were coded as events were not necessarily matched in each subject. If, in one particular subject, all the correct remote trials coded as events were scored 3 and all the correct recent trials coded as events were scored 2, then the retrieval success effect, for example, which is expected to correlate with the confidence level, should be confounded in the remote trials. To incorporate the individual difference related to the confidence level unmatched between the remote and recent trials, the confidence difference (CD) was defined as follows: $\mathrm{CD}=N_{\mathrm{Rem} 3} /\left(N_{\mathrm{Rem} 3}+N_{\mathrm{Rem} 2}\right)-N_{\mathrm{Rec} 3} /\left(N_{\mathrm{Rec} 3}+\right.$ $N_{\text {Rec } 2}$ ), where $N_{\text {Rem } 3}, N_{\text {Rem2 } 2}, N_{\text {Rec3 }}$, and $N_{\text {Rec } 2}$ represent the number of correct remote trials with confidence score 3 , the number of correct remote trials with confidence score 2 , the number of correct recent trials with confidence score 3 , and the number of correct recent trials with confidence score 2 , respectively. The $\mathrm{CD}$ reflects the difference in the proportion of high confidence remote trials versus the proportion of high confidence recent trials. So, if all the correct remote trials coded as events were scored 3 and all the correct recent trials coded as events were scored 2 in one subject, then the subject should be more confident in remote trials and the $\mathrm{CD}$ equals to 1 .

The contrast images for remote versus recent trials from each subject were entered into a second-level group analysis. Only the correct trials that also received 2 or 3 confidence score were used to generate the contrast images to enhance memory related signals. The group analyses were conducted using a random effect model, with a single correlation of the $\mathrm{CD}$ : The constant regressor represents the effect of remote versus recent trials, whereas the correlation regressor represents the effect of the 


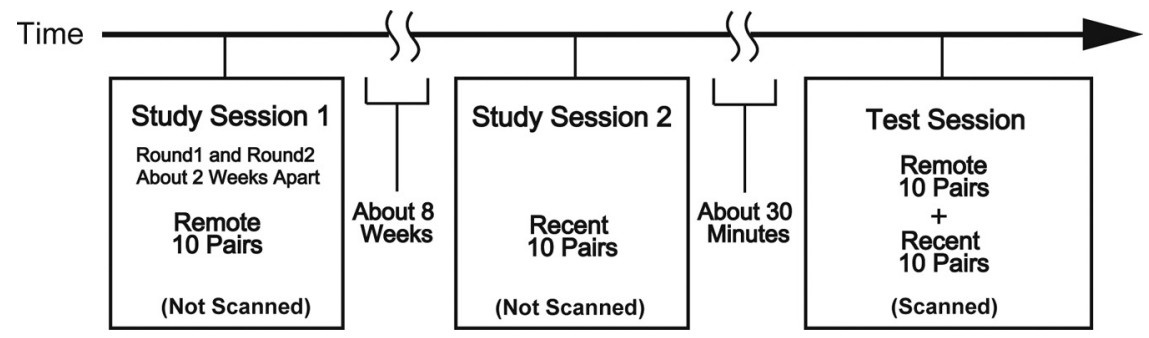

Figure 2. Time schedule for detection of long-term memory formation. The subjects first learned 10 paired figures in session 1 , and after $\sim 8$ weeks, learned another 10 pairs in session 2 . Then fMRI test session was administered.

confidence level. Significant activations were detected using a small volume correction based on the estimated volume of the gray matter in the bilateral MTL, i.e., the hippocampi, and Brodmann areas (BA) 28, 34, 35, and $36\left(20 \mathrm{~cm}^{3}\right)$, and the gray matter in the bilateral temporal neocortex, i.e., BA 20, 21, 37, and $38\left(50 \mathrm{~cm}^{3}\right)$ (Maldjian et al., 2003).

\section{Results}

\section{Behavioral data}

The interval between R1 and R2 in S1 was $16.2 \pm 4.8 \mathrm{~d}$ (mean \pm $\mathrm{SD})$, and the interval between R2 in S1 and S2 was $62.6 \pm 10.0 \mathrm{~d}$. The numbers of trials taken to achieve the criteria for the remote pairs in $\mathrm{S} 1$ were $14.4 \pm 4.2($ mean $\pm \mathrm{SD})$ and $8.3 \pm 1.7$ trials in $\mathrm{R} 1$ and $\mathrm{R} 2$, respectively, and the number of trials taken to achieve the criteria for the recent pairs in S2 was $3.5 \pm 1.5$ trials.

The correct performance of the mid-session retention test for the pairs learned in $\mathrm{R} 1$ in S1, which was administered just before $\mathrm{R} 2$ in $\mathrm{S} 1$, was $76.0 \pm 3.7 \%$ (mean $\pm \mathrm{SEM}$ ), and the reaction time in correct trials was $1176 \pm 43 \mathrm{~ms}$. In contrast, the correct performance of the test session, which was administered in the scanner, was $71.3 \pm 3.3 \%$ in remote trials and $79.3 \pm 2.8 \%$ in recent trials, and these were not significantly different. The reaction time in correct trials was $1219 \pm 38 \mathrm{~ms}$ in remote trials and $1158 \pm 36 \mathrm{~ms}$ in recent trials, and these were not significantly different, either. Moreover, there was no significant difference in correct performance and reaction time between remote trials in the test session and all the trials in the mid-session retention test.

The confidence scores collected after the scan were compatible with the percentage of correct performance in remote and recent trials. In remote trials, the percentage of correct trials out of those trials that received score 1 was $54.2 \pm 10.3 \%$ (mean \pm SEM), and the percentage of correct trials out of those trials that received score 2 or 3 was $73.6 \pm 3.4 \%$, and these were significantly different $\left[t_{(48)}=\right.$ $2.1, p<0.05]$. Similarly, in recent trials, the percentage of correct trials out of the score- 1 trials was $65.5 \pm 7.5 \%$, and the percentage of correct trials out of the score- 2 or -3 trials was $80.9 \pm 2.9 \%$, and these were significantly different $\left(t_{(50)}=2.1, p<0.05\right)$. These results validate the procedures of using only the correct trials that received confidence score 2 or 3 to generate the contrast images of remote versus recent trials. Moreover, in remote trials, the percentage of correct trials out of the score- 2 trials was $64.4 \pm 5.3 \%$, and the percentage of correct trials out of the score- 3 trials was $84.0 \pm 3.7 \%$, and these were also significantly different $\left(t_{(55)}=\right.$ $3.0, p<0.005)$. Similarly, in recent trials, the percentage of correct trials out of the score- 2 trials was $71.5 \pm 6.1 \%$, and the percentage of correct trials out of the score- 3 trials was $87.0 \pm$ $4.0 \%$, and these were also significantly different $\left(t_{(51)}=2.2, p<\right.$ $0.05)$. These results validate the definition of the CD based on the difference between the score- 3 trials and the score- 2 trials. Additionally, there was no significant difference between the percentage of correct trials out of the score- 1 and score- 2 trials in remote or recent trials.
The confidence scores were used for calculation of the contrast of remote versus recent trials. The percentage of the trials in the test session used for the imaging analyses (i.e., the correct trials that also received the confidence score 2 or 3 ) was $62.3 \pm 3.7 \%$ (mean $\pm \mathrm{SEM})$ in remote trials and $65.3 \pm 4.0 \%$ in recent trials, and these were not significantly different. The reaction time in these trials was $1198 \pm 39$ $\mathrm{ms}$ in remote trials and $1130 \pm 35 \mathrm{~ms}$ in recent trials, and these were not significantly different, either.

\section{Imaging data}

A group analysis was conducted using a simple correlation that contained a constant regressor and a correlation regressor (see Materials and Methods). The constant regressor, which is of central interest of this study, reflects remote versus recent trials, and was calculated based on the correct trials that also received confidence score 2 or 3 (Fig. 3). The posterior part of the hippocampus in the right hemisphere at $(26,-38,0)$ elicited greater brain activity in recent trials than in remote trials $(t=3.6, p<0.05$ SVC) (Fig. 3A). In contrast, the anterior temporal region in the left hemisphere at $(-28,14,-26)$ elicited greater brain activity in remote trials than in recent trials $(t=4.4, p<0.05$ SVC). The single voxel in the anterior temporal region in the right hemisphere at $(28,14,-26)$ that corresponds to the peak voxel in the left anterior temporal activation also elicited greater brain activity in remote trials $(t=3.4)$, although its peak was not significant after SVC. No significant brain activity was observed outside the anterior temporal cortex above uncorrected $p<0.001$ or after correction of whole-brain multiple comparisons.

The left anterior temporal activation was examined further, by using across-individual variability of the reaction time difference between remote trials in the test session and all the trials in the mid-session retention test. The same pairs of the Fourier figures were presented in the test session and the mid-session retention test using the same task structures and time parameters, but $\sim 8$ and 2 weeks after learning, respectively. The percentage of correct performance and the reaction time in these two tests were not significantly different (see Behavioral data). We reasoned that, if the anterior temporal activation reflected consolidated memory contents, then the memory trace should help to choose the answer efficiently and the reaction time should decrease as a result. More specifically, the greater the anterior temporal activation that reflected the stable long-term memory representation, the shorter the reaction time in remote trials in the test session relative to that in the mid-session retention test. Only trials that were correct in both of the two tests were included in this analysis. Figure $3 B$ demonstrates significant correlation in the left anterior temporal region $(r=0.53, p<0.005)$, with shortened reaction time difference correlated with greater brain activity.

An additional analysis was made on the correlation regressor in a random effect model that reflects the effect of the confidence level. Figure $4 A$ shows the significant correlation observed in multiple brain regions including the right hippocampal region $(22,-30,-8)(t=4.8, p<0.05 \mathrm{FDR}$ whole-brain corrected $)$ and the left parietal region at $(-56,-36,50)(t=4.8, p<0.05 \mathrm{FDR}$ whole-brain corrected). The scatter plots in the hippocampal and parietal regions demonstrate that the greater the $\mathrm{CD}$ (i.e., the more confident on remote trials), the greater the signal in remote trials, and conversely, the smaller the CD (i.e., the more confident 


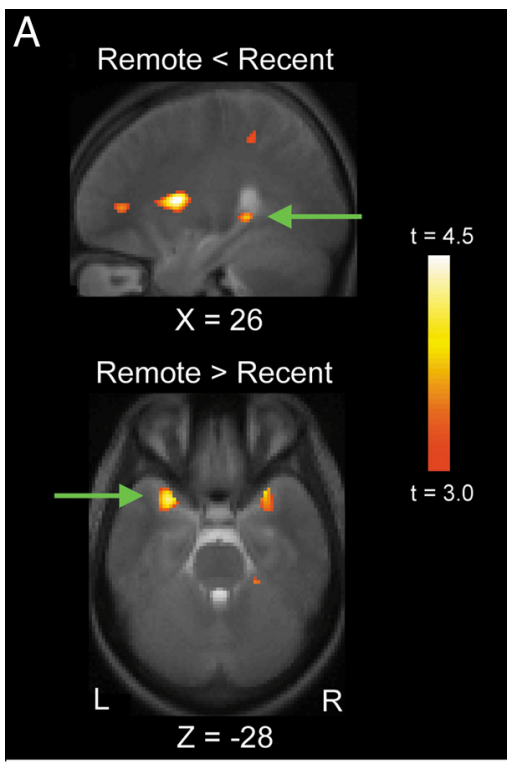

B

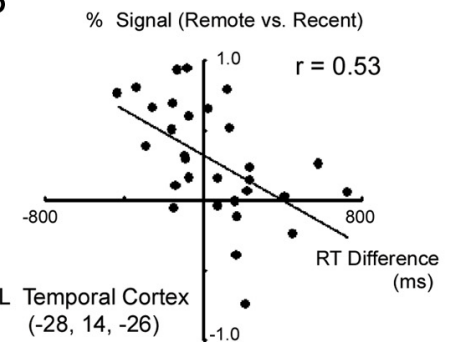

Figure 3. A, Statistical activation map for the contrast of recent versus remote trials (top) and remote versus recent trials (bottom). The activation map is displayed as a sagittal or transverse section and is overlaid on top of the anatomic image averaged across subjects. Significant activations in the right hippocampal region (top) and the left anterior temporal region (bottom) are indicated by green arrows. Statistical significance is indicated using the color scale to the right, and the section level is indicated using the $X$ or $Z$ coordinate of the Talairach space. $B$, Scatter plots of the anterior temporal activation against reaction time difference between remote trials in the test session and all the trials in the mid-session retention test (see Materials and Methods). One plot indicates data from one subject. L, Left; $R$, right.

on recent trials), the greater the signal in recent trials (Fig. $4 B$ ). We also examined to what degree the hippocampal region of interest (ROI) revealed by the confidence level correlation analysis $(22,-30,-8)$ was the same or different than the ROI revealed by the recent $>$ remote analysis $(26,-38,0)$. The region revealed by the confidence level correlation analysis $(22,-30$, -8 ) did not show significant signal difference in the recent $>$ remote analysis $(t=-0.9, p>0.05)$, suggesting one dissociable aspect of these regions. Conversely, the region revealed by the recent $>$ remote analysis $(26,-38,0)$ showed significant correlation in the confidence level correlation analysis $(t=2.4, p<$ $0.05)$, similarly to the other region.

Finally, basic contrasts were calculated to compare activation with previous fMRI studies of paired associate retrieval (Jackson and Schacter, 2004; Kirwan and Stark, 2004; Ranganath et al., 2004; Tanabe et al., 2005; Lewis-Peacock and Postle, 2008). Specifically, remote + recent trials were contrasted against fixation baseline (supplemental Fig. S1, available at www.jneurosci.org as supplemental material). Compared with fixation baseline, many regions were activated. Occipital regions were activated in response to the visual demands of the task, and the left motor cortex and supplementary motor cortex were activated, presumably because of the demand of making a button press. Outside these

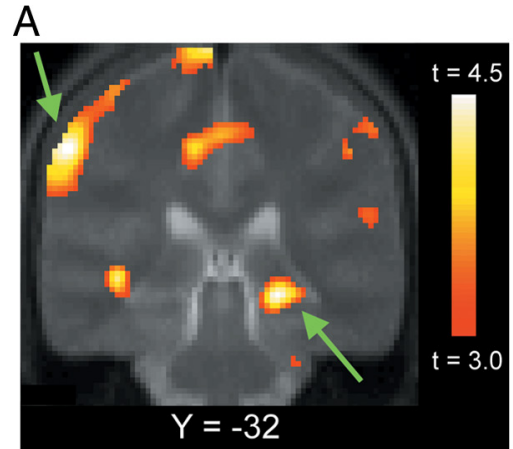

B

$\%$ Signal (Remote vs. Recent)

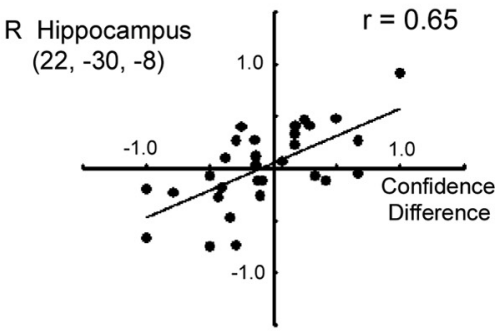

$\%$ Signal (Remote vs. Recent)

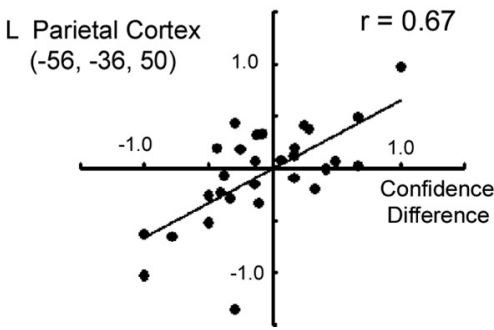

Figure 4. A, Statistical maps for the effect of the confidence level during retrieval independent of trial types remote/recent. The format is similar to Figure $3 A$. The right hippocampal region and the left parietal region are pointed by green arrows. $\boldsymbol{B}$, Scatter plots of the activations in the right hippocampal and the left parietal regions against confidence difference (see Materials and Methods for details). The format is similar to Figure 3B. L, Left; R, right.

sensory/motor regions, activated regions were also observed in many areas of the frontal and parietal regions, basal ganglia and thalamus. Although the precise functional characterization of the regions identified by this contrast provides little insight into specific correlates of paired associate retrieval, this activation pattern is similar to that observed in previous paired associate retrieval tasks.

\section{Discussion}

In the present fMRI study, retrieval of initially learned figures was compared with retrieval of newly learned figures. The right hippocampal region elicited greater brain activity during retrieval of newly learned figures than retrieval of initially learned figures. In contrast, the left anterior temporal region showed the opposite activation pattern. These results suggest that the cortical substrates for enduring memory representation can be experimentally created in the temporal neocortex.

Although the different roles of the hippocampus have been proposed by different theories of long-term memory formation (Gaffan and Gaffan, 1991; Squire and Alvarez, 1995; Nadel and Moscovitch, 1997; Rolls, 2000), hippocampal activity is typically observed during memory encoding in the context of subsequent memory effects (Wagner et al., 1998a; Otten et al., 2001; Ofen et al., 2007) or immediately after memory encoding (Ranganath 
and D'Esposito, 2001) in human neuroimaging studies. The hippocampus, as well as the parietal cortex, is also known to be activated during retrieval success (Henson et al., 1999; Eldridge et al., 2000; Konishi et al., 2000; Yonelinas, 2002; Manns et al., 2003), consistent with the results of the hippocampal and parietal activation in the present study that correlated with the level of confidence. The hippocampal involvement has also been shown to disappear in the passage of time (Squire and Alvarez, 1995), as revealed by decreasing hippocampal activity during retrieval of encoded information (Takashima et al., 2006; Smith and Squire, 2009). Consistent with the previous studies, our results demonstrated smaller brain activity in the hippocampal region during retrieval of initially learned figures than during retrieval of newly learned figures.

The present study demonstrated the long-term representation for unfamiliar figures in the temporal neocortex. There are several previous neuroimaging studies that attempted to show the shift of retrieval mechanisms from the hippocampus to the temporal neocortex (Stark and Squire, 2000; Takashima et al., 2006). Although it was shown that the activity in the hippocampus decreased with the passage of time, consistent with the time-limited role of the hippocampus, these studies did not show temporal neocortical representation that developed after learning. One notable exception is reported by Takashima et al. (2007), where similar anterior temporal activation was observed during retrieval of remote (stabilized) pairs relative to retrieval of recent (labile) pairs. The anterior temporal activation reported in the study by Takashima et al. (2007) was located in the lateral part of the anterior temporal cortex $(\sim \pm 50$ in $x$-coordinate $)$, whereas the anterior temporal activation reported in this study was located more medially ( $\sim \pm 30$ in $x$-coordinate). One possible explanation for the different results would be that the remote pairs in Takashima et al. (2007) were studied for 1 week and scanning was conducted $1 \mathrm{~d}$ after the end of the study, whereas the remote pairs in this study were studied $\sim 8$ weeks before scanning. Thus, it is possible that localization of long-term representations formed in the anterior temporal cortex depends on how much time has passed since the memory content was encoded.

The anterior temporal activation during retrieval of initially learned figures observed in the present study is consistent with past studies, in terms of both localization and laterality. The anterior temporal activation converges with the results of neuropsychological studies showing permanent retrograde amnesia following anterior temporal cortical damage and with the results of neuroimaging studies on semantic knowledge (Kapur et al., 1992; Damasio et al., 1996; Gorno-Tempini et al., 1998; Cabeza and Nyberg, 2000; Bar et al., 2001; Martin and Chao, 2001; Tsukiura et al., 2002; Thaiss and Petrides, 2003; Ploran et al., 2007). Although the right hippocampal activation during retrieval of newly learned figures followed the rule of the right hemisphere dominance in nonverbal processing (Milner, 1971; Kelley et al., 1998; Wagner et al., 1998b; Gazzaniga, 2005), the anterior temporal region did not. However, the final memory representation can be formed primarily in the left hemisphere even for nonverbal items, as demonstrated by the results of a previous study showing that the anterior temporal cortex in the left hemisphere is involved in retrieval of familiar names whereas the anterior temporal cortex in the right hemisphere is involved in retrieval of newly learned names (Tsukiura et al., 2002).

The memory representation for the remote and recent pairs might not be the same kind. The temporopolar cortex may form configural representation that supports memory retrieval based on familiarity after repeated exposures to the pair (Haskins et al.,
2008). However, the hippocampus encodes relational representation that supports conscious recollection of recent events. Although the present results cannot discriminate whether the anterior temporal activation reflects the consolidated memory representation that is similar to the one encoded into the hippocampus, or configural representation based on familiarity, the present results demonstrate the formation of long-term representation in the anterior temporal cortex.

The temporal neocortical region was more activated when older memory had to be retrieved. The results may be parallel to our previous monkey electrophysiological study of the PA task using the same Fourier figures that the monkeys had never seen before. Thus, the stimulus-selective activity in the IT neuron in our electrophysiological study (Miyashita, 1988; Sakai and Miyashita, 1991) may correlate with the temporal cortical activation during retrieval of the initially learned figures in this study. Although more investigation is required to elucidate the precise role of the anterior temporal cortex, the present fMRI study, collectively with previous neuropsychological studies of retrograde amnesia (Kapur et al., 1992; Tsukiura et al., 2002), provides converging evidence for stable memory representation developed in the anterior temporal cortex.

\section{References}

Bar M, Tootell RB, Schacter DL, Greve DN, Fischl B, Mendola JD, Rosen BR, Dale AM (2001) Cortical mechanisms specific to explicit visual object recognition. Neuron 29:529-535.

Cabeza R, Nyberg L (2000) Imaging cognition II: An empirical review of 275 PET and fMRI studies. J Cogn Neurosci 12:1-47.

Damasio H, Grabowski TJ, Tranel D, Hichwa RD, Damasio AR (1996) A neural basis for lexical retrieval. Nature 380:499-505.

Eldridge LL, Knowlton BJ, Furmanski CS, Bookheimer SY, Engel SA (2000) Remembering episodes: a selective role for the hippocampus during retrieval. Nat Neurosci 3:1149-1152.

Gaffan D, Gaffan EA (1991) Amnesia in man following transection of the fornix. A review. Brain 114:2611-2618.

Gais S, Albouy G, Boly M, Dang-Vu TT, Darsaud A, Desseilles M, Rauchs G, Schabus M, Sterpenich V, Vandewalle G, Maquet P, Peigneux P (2007) Sleep transforms the cerebral trace of declarative memories. Proc Natl Acad Sci U S A 104:18778-18783.

Gazzaniga MS (2005) Forty-five years of split-brain research and still going strong. Nat Rev Neurosci 6:653-659.

Gorno-Tempini ML, Price CJ, Josephs O, Vandenberghe R, Cappa SF, Kapur N, Frackowiak RS, Tempini ML (1998) The neural systems sustaining face and proper-name processing. Brain 121:2103-2118.

Haist F, Bowden Gore J, Mao H (2001) Consolidation of human memory over decades revealed by functional magnetic resonance imaging. Nat Neurosci 4:1139-1145.

Haskins AL, Yonelinas AP, Quamme JR, Ranganath C (2008) Perirhinal cortex supports encoding and familiarity-based recognition of novel associations. Neuron 59:554-560.

Henson RN, Rugg MD, Shallice T, Josephs O, Dolan RJ (1999) Recollection and familiarity in recognition memory: an event-related functional magnetic resonance imaging study. J Neurosci 19:3962-3972.

Higuchi S, Miyashita Y (1996) Formation of mnemonic neuronal responses to visual paired associates in inferotemporal cortex is inpaired by perirhinal and entorhinal lesions. Proc Natl Acad Sci U S A 93:739-743.

Jackson O 3rd, Schacter DL (2004) Encoding activity in anterior medial temporal lobe supports subsequent associative recognition. Neuroimage 21:456-462.

Kapur N, Ellison D, Smith MP, McLellan DL, Burrows EH (1992) Focal retrograde amnesia following bilateral temporal lobe pathology. A neuropsychological and magnetic resonance study. Brain 115:73-85.

Kelley WM, Miezin FM, McDermott KB, Buckner RL, Raichle ME, Cohen NJ, Ollinger JM, Akbudak E, Conturo TE, Snyder AZ, Petersen SE (1998) Hemispheric specialization in human dorsal frontal cortex and medial temporal lobe for verbal and nonverbal memory encoding. Neuron 20:927-936. 
Kirwan CB, Stark CE (2004) Medial temporal lobe activation during encoding and retrieval of novel face-name pairs. Hippocampus 14:919-930.

Konishi S, Wheeler ME, Donaldson DI, Buckner RL (2000) Neural correlates of episodic retrieval success. Neuroimage 12:276-286.

Lewis-Peacock JA, Postle BR (2008) Temporary activation of long-term memory supports working memory. J Neurosci 28:8765-8771.

Maldjian JA, Laurienti PJ, Kraft RA, Burdette JH (2003) An automated method for neuroanatomic and cytoarchitectonic atlas-based interrogation of fMRI data sets. Neuroimage 19:1233-1239.

Manns JR, Hopkins RO, Reed JM, Kitchener EG, Squire LR (2003) Recognition memory and the human hippocampus. Neuron 37:171-180.

Martin A, Chao LL (2001) Semantic memory and the brain: structure and processes. Curr Opin Neurobiol 11:194-201.

Messinger A, Squire LR, Zola SM, Albright TD (2001) Neuronal representations of stimulus associations develop in the temporal lobe during learning. Proc Natl Acad Sci U S A 98:12239-12244.

Milner B (1971) Interhemispheric differences in the localization of psychological processes in man. Br Med Bull 27:272-277.

Milner B, Corkin S, Teuber HL (1968) Further analysis of the hippocampal amnesic syndrome: 14-year follow-up study of H.M. Neuropsychologia $6: 215-234$.

Mishkin M, Murray EA (1994) Stimulus recognition. Curr Opin Neurobiol 4:200-206.

Miyashita Y (1988) Neuronal correlate of visual associative long-term memory in the primate temporal cortex. Nature 335:817-820.

Nadel L, Moscovitch M (1997) Memory consolidation, retrograde amnesia and the hippocampal complex. Curr Opin Neurobiol 7:217-227.

Naya Y, Yoshida M, Miyashita Y (2003) Forward processing of long-term associative memory in monkey inferotemporal cortex. J Neurosci 23:2861-2871.

Ofen N, Kao YC, Sokol-Hessner P, Kim H, Whitfield-Gabrieli S, Gabrieli JD (2007) Development of the declarative memory system in the human brain. Nat Neurosci 10:1198-1205.

Otten LJ, Henson RN, Rugg MD (2001) Depth of processing effects on neural correlates of memory encoding: relationship between findings from across- and within-task comparisons. Brain 124:399-412.

Plihal W, Born J (1997) Effects of early and late nocturnal sleep on declarative and procedural memory. J Cogn Neurosci 9:534-547.

Ploran EJ, Nelson SM, Velanova K, Donaldson DI, Petersen SE, Wheeler ME (2007) Evidence accumulation and the moment of recognition: dissociating perceptual recognition processes using fMRI. J Neurosci 27:11912-11924.

Ranganath C, D’Esposito M (2001) Medial temporal lobe activity associated with active maintenance of novel information. Neuron 31:865-873.

Ranganath C, Cohen MX, Dam C, D'Esposito M (2004) Inferior temporal, prefrontal, and hippocampal contributions to visual working memory maintenance and associative memory retrieval. J Neurosci 24:3917-3925.

Rolls ET (2000) Memory systems in the brain. Annu Rev Psychol 51: 599-630.
Sakai K, Miyashita Y (1991) Neural organization for the long-term memory of paired associates. Nature 354:152-155.

Scoville WB, Milner B (1957) Loss of recent memory after bilateral hippocampal lesions. J Neurol Neurosurg Psychiatry 20:11-21.

Smith CN, Squire LR (2009) Medial temporal lobe activity during retrieval of semantic memory is related to the age of the memory. J Neurosci 29:930-938.

Squire LR, Alvarez P (1995) Retrograde amnesia and memory consolidation: a neurobiological perspective. Curr Opin Neurobiol 5:169-177.

Stark CE, Squire LR (2000) fMRI activity in the medial temporal lobe during recognition memory as a function of study-test interval. Hippocampus 10:329-337.

Takashima A, Petersson KM, Rutters F, Tendolkar I, Jensen O, Zwarts MJ, McNaughton BL, Fernández G (2006) Declarative memory consolidation in humans: a prospective functional magnetic resonance imaging study. Proc Natl Acad Sci U S A 103:756-761.

Takashima A, Nieuwenhuis IL, Rijpkema M, Petersson KM, Jensen O, Fernández G (2007) Memory trace stabilization leads to large-scale changes in the retrieval network: a functional MRI study on associative memory. Learn Mem 14:472-479.

Tanabe HC, Honda M, Sadato N (2005) Functionally segregated neural substrates for arbitrary audiovisual paired-association learning. J Neurosci 25:6409-6418.

Thaiss L, Petrides M (2003) Source versus content memory in patients with a unilateral frontal cortex or a temporal lobe excision. Brain 126: $1112-1126$.

Tsukiura T, Fujii T, Fukatsu R, Otsuki T, Okuda J, Umetsu A, Suzuki K, Tabuchi M, Yanagawa I, Nagasaka T, Kawashima R, Fukuda H, Takahashi S, Yamadori A (2002) Neural basis of the retrieval of people's names: evidence from brain-damaged patients and fMRI. J Cogn Neurosci 14:922-937.

Wagner AD, Schacter DL, Rotte M, Koutstaal W, Maril A, Dale AM, Rosen BR, Buckner RL (1998a) Building memories: remembering and forgetting of verbal experiences as predicted by brain activity. Science 281:1188-1191.

Wagner AD, Poldrack RA, Eldridge LL, Desmond JE, Glover GH, Gabrieli JD (1998b) Material-specific lateralization of prefrontal activation during episodic encoding and retrieval. Neuroreport 9:3711-3717.

Worsley KJ, Friston KJ (1995) Analysis of fMRI time-series revisited-again. Neuroimage 2:173-181.

Yonelinas AP (2002) The nature of recollection and familiarity: a review of 30 years of research. J Mem Lang 46:441-517.

Yoo SS, Hu PT, Gujar N, Jolesz FA, Walker MP (2007) A deficit in the ability to form new human memories without sleep. Nat Neurosci 10:385-392.

Zola-Morgan S, Squire LR, Amaral DG (1986) Human amnesia and the medial temporal region: enduring memory impairment following a bilateral lesion limited to field CA1 of the hippocampus. J Neurosci 6:2950-2967.

Zola-Morgan SM, Squire LR (1990) The primate hippocampal formation: evidence for a time-limited role in memory storage. Science 250:288 -290. 